\title{
PERFIL ANTROPOMÉTRICO DE MULHERES ADULTAS DA ÁREA URBANA E RURAL NO MUNICÍPIO DE BARCELOS, AM.
}

\author{
Dionísia NAGAHAMA, Lúcia Kiyoko Ozaki YUYAMA, \\ Fernando Hélio ALENCAR
}

\begin{abstract}
RESUMO - De um total de 203 mulheres adultas entrevistadas, foram avaliadas 175 (entre 16 a 73 anos), por meio de medidas antropométricas: peso, altura, prega cutânea tricipital (PCT), circunferência braquial (CB), circunferência muscular (CMB) e Índice de Massa Corpórea (IMC). Das mulheres analisadas, $84,0 \%(\mathrm{n}=147)$ residiam na zona urbana e $16,0 \%(\mathrm{n}=28)$ na zona rural. Verificou-se que o peso médio das mulheres não nutrizes residentes na área urbana foi de $54,1 \mathrm{~kg}$; altura de $149,0 \mathrm{~cm}$ e o IMC de $24,4 \mathrm{~kg} / \mathrm{m}^{2}$, enquanto que a PCT, CB e a CMB forneceram valores médios de $19,2 \mathrm{~mm}, 27,5 \mathrm{~cm}$ e $21,5 \mathrm{~cm}$, respectivamente. $\mathrm{Na}$ área rural, as mulheres apresentaram um peso médio de $50,4 \mathrm{~kg}$, altura de $148,6 \mathrm{~cm}, \mathrm{IMC}$ de $23,0 \mathrm{~kg} / \mathrm{cm}^{2}$ e a PCT, CB e a CMB foram $13,8 \mathrm{~mm}, 26,4 \mathrm{~cm}$ e $22,1 \mathrm{~cm}$, respectivamente. Houve diferença estatisticamente significativa apenas nos valores médios da PCT entre as mulheres procedentes da área urbana e rural $(\mathrm{p}<0,05)$. Nas mulheres da área urbana, a ocorrência de baixo peso foi de $6,5 \%, 28,2 \%$ de sobrepeso e $11,3 \%$ de obesidade. Nas mulheres da área rural, a prevalência de baixo peso e obesidade foi de $4 \%$ e de sobrepeso de $16 \%$. A maioria da população estudada situou-se no limite máximo de normalidade do IMC, e os índices representantes das reservas adiposas (PCT) foram maiores nas mulheres residentes na área urbana.
\end{abstract}

Palavras-chave: Avaliação antropométrica, mulheres, obesidade.

\section{Anthropometric profile of women from the urban and rural areas in Barcelos municipality. (Amazonas, Brazil).}

\begin{abstract}
From a total of 203 adult women interviewed, 175 subjects aged 16 to 73 years were evaluated for the anthropometrics variables: weight, height, triceps skinfold thickness, midupper arm circumference, arm muscle circumference and body mass index (BMI). Of these women, $84,0 \%$ (147) were living in the urban area and 16,0\% (28) in the rural area. Observing the mean values for non-lactating women in the urban area were: weight $54,1 \mathrm{~kg}$, height $149,0 \mathrm{~cm}$, BMI $24,4 \mathrm{~kg} / \mathrm{m}^{2}$, triceps skinfold thickness $19,2 \mathrm{~mm}$, arm circumference $27,5 \mathrm{~cm}$, arm muscle circumference $21,5 \mathrm{~cm}$. For women in the rural area the values were: weight $50,4 \mathrm{~kg}$, height $148,6 \mathrm{~cm}$, BMI $23,0 \mathrm{~kg} / \mathrm{cm}^{2}$, triceps skinfold thickness $13,8 \mathrm{~mm}$, arm circumference $26,4 \mathrm{~cm}$, arm muscle circumference $22,1 \mathrm{~cm}$. There was a significant difference in the average triceps skinfold thickness values between women in the rural area and women in the urban area $(p<0,05)$. In the urban area women a $6,5 \%$ prevalence of low weight was found, a $28,2 \%$ prevalence of overweight and a $11,3 \%$ prevalence of obesity. In the rural area women low weight and obesity were found in $4 \%$, and overweight in $16 \%$. In this study, most women were at the upper limit of normality for BMI, with the indices of fat reserves being highest in the women of the urban area.
\end{abstract}

Key-words: anthropometry, women, obesity.

\section{INTRODUÇÃO}

O Amazonas é o maior Estado do Brasil com características geográficas que dificultam o acesso às áreas mais distantes, como em zonas rurais, onde a principal via de acesso é a fluvial. Possivelmente em decorrência destes fatores, a literatura regional apresenta estudos

${ }^{1}$ Instituto Nacional de Pesquisas da Amazônia - INPA/CPCS. - CP. 478 Manaus, AM. CEP 69083-000 
relativamente escassos sobre as condições de vida, saúde e nutrição dos diferentes segmentos e grupos populacionais da Amazônia, a maior parte destas pesquisas referem-se à população urbana de Manaus (Shrimpton,1984; Lehti, 1989; Yuyama \& Nagahama,1992; Nagahama et al., 1990), sendo raros e fragmentados os estudos sobre as populações do interior do Estado (Diniz,1991; Yuyama et al., 1999; Alencar et al., 2000). Alem disso, os estudos de maior expressão epidemiológica foram realizados há aproximadamente duas décadas (Giugliano et al.,1981; 1984).

É notório que a sociedade moderna tem vivenciado um processo de transformação no estilo de vida, influenciando decisivamente o padrão de saúde e nutrição da população. Como conseqüência, observa-se o desencadeamento de processos carenciais, expressos pelo agravamento da curva de prevalência, tanto da obesidade como dos casos de desnutrição energético-protéica (Coitinho et al., 1991). Portanto, estudos de avaliação do estado nutricional de grupos populacionais têm sua importância epidemiológica, tanto para a viabilização do diagnóstico da desnutrição e/ou obesidade, como para implantação de programas de recuperação e prevenção (Costa et al., 1987).

No Brasil há poucos estudos referentes ao perfil antropométrico de mulheres adultas, sendo os de maior abrangência nacional aqueles realizados pelo ENDEF-1974/75 e PNSN (Coitinho et al.,1991). Outras pesquisas de menor abrangência foram realizadas em diferentes regiões (Faintuch et al., 1983; Campana et al., 1987; Costa et al., 1987; Anselmo et al., 1992; Ell et al., 1999; Martins, et al., 1999). Estes estudos, apesar de pontuais, são úteis para o estabelecimento de valores de referência regionais e para a comparação com outros grupos de características sócioeconômicas similares. Na Amazônia Brasileira os trabalhos disponíveis para traçar um perfil nutricional de mulheres adultas são escassos e desenvolvidos com pequenos grupos populacionais, de área urbana (Guedes, 1989; Yuyama \& Nagahama, 1992; SEMSA/UNICEF, 1996; Oliveira et al., 1998) e rural (Silva, 1959; Giugliano et al., 1981; 1984).

Em decorrência da escassez destas informações, o presente trabalho teve como objetivo descrever o perfil antropométrico de mulheres adultas residentes nas áreas urbana $\mathrm{e}$ rural do Município de Barcelos-AM, contribuindo assim para a determinaçao do estado nutricional das populaçoes da Amazônia.

\section{MATERIAL E MÉTODOS}

\section{Local do Estudo}

Barcelos localiza-se ao Norte do Estado do Amazonas, situando-se na margem direita do Rio Negro, afluente do Rio Amazonas, a 40 $\mathrm{m}$ acima do nível do mar, distando da capital do Estado, $396 \mathrm{~km}$ em linha reta e $496 \mathrm{~km}$ por via fluvial, é o maior município do Estado do Amazonas, com uma área de $89.572 \mathrm{~km}^{2}$ e uma população de 10.856 habitantes, o que configura uma densidade demográfica de 0,12 hab./ km². (SEBRAE-AM, 1994).

\section{População Estudada}

A coleta de dados foi realizada no período de outubro de 1995 a fevereiro de 1996, sendo parte integrante de um projeto multidisciplinar que investiga as condições de saúde e nutrição das populações dos diferentes ecossistemas do Amazonas.

Participaram da pesquisa 175 mulheres com idades compreendidas entre 16 e 73 anos, residentes na área rural e urbana, cuja participação foi por livre demanda, bem como um grupo de 26 nutrizes, sendo 23 da área urbana e 3 da área rural, do referido Município.

\section{Medidas}

Trata-se de um estudo transversal, observacional e descritivo, onde se avaliou o estado nutricional da população por meio de medidas antropométricas: peso, altura, prega cutânea tricipital (PCT) e circunferência braquial (CB), obtidas de acordo com as recomendações estabelecidas por Jelliffe (1966). Para o peso utilizou-se uma balança mecânica com capacidade para $150 \mathrm{~kg}$ e divisão de $100 \mathrm{~g}$, estando as mulheres com roupas leves e sem sapatos. A altura foi medida utilizando-se um antropômetro metálico afixado em parede sem rodapé, com visor de plástico, esquadro e nivelador na sua extremidade. A PCT foi obtida utilizando-se um paquímetro marca CrymmychHoltain Ltd. (UK), com pressão constante de $10 \mathrm{~g} / \mathrm{mm}^{2}$ e precisão de $0,2 \mathrm{~mm}$, onde foram 
realizadas três mensurações consecutivas, tomando-se a média das medidas; a CB foi medida com fita métrica extensível marca Centiverâ (França) com precisão de $0,1 \mathrm{~cm}$. Foram calculados os valores do Índice de Massa Corpórea (IMC) e circunferência muscular do braço (CMB). A partir do IMC ou o Índice de Quetelet, que representa a composição corpórea através das medidas de peso e altura, seguiu-se a classificação proposta pela OMS (1995): Baixo peso $\left(<18,5 \mathrm{~kg} / \mathrm{m}^{2}\right)$, Normal $(18,5-$ $\left.24,99 \mathrm{~kg} / \mathrm{m}^{2}\right)$, Sobrepeso $\left(25-29,99 \mathrm{~kg} / \mathrm{m}^{2}\right)$ e Obeso $\left(>30 \mathrm{~kg} / \mathrm{m}^{2}\right)$. Com relação ao cálculo da $\mathrm{CMB}$, utilizou-se a seguinte fórmula: $\mathrm{CMB}=$ $\mathrm{CB}-(3,14 \times \mathrm{PCT})$. Todas as medidas foram tomadas por um único pesquisador.

As análises estatísticas foram realizadas utilizando-se o programa EPI INFO, versão 6.02, obtendo-se a análise descritiva das medidas de tendência central e de dispersão. Empregou-se o teste t-Student ou análise de variância para a comparação dos valores médios das variáveis contínuas. O nível de rejeição da hipótese de nulidade fixado foi menor ou igual a $5 \%(\mathrm{p}<0,05)$.

\section{RESULTADOS}

Das 175 mulheres incluídas no estudo, $84,0 \%$ residiam na área urbana e $16,0 \%$ na área rural. No universo estudado, houve maior representação das faixas etárias compreendidas entre 20 e 29 anos $(36,6 \%)$ e dos 30 a 39 anos $(32,6 \%)$ (Tabela 1). Foram incluídas 6 idosas (> 60 anos), sendo uma da área rural e 5 da área urbana, alem de 10 adolescentes ( $<20$ anos), 1 da área rural e 9 da área urbana.

Nas mulheres da área urbana, a analise das medidas antropométricas, mostrou uma tendência decrescente dos valores médios da PCT com o decorrer da idade (Tabela 1$)$. Houve diferença significativa $(p<0,05)$ entre os valores de PCT das mulheres da zona urbana $(19,2+$ $7,0 \mathrm{~mm})$ e rural $(13,8+5,2 \mathrm{~mm})$, o mesmo não ocorrendo com os demais valores antropométricos.

Devido às modificações do peso e da composição do organismo decorrentes da lactação, os resultados antropométricos das nutrizes são apresentados a parte. Optou-se pela junção das duas populações em virtude do pequeno número amostral para a realização de análises estatísticas. Verifica-se que os valores não diferem das médias antropométricas das mulheres não nutrizes, excetuando a média da PCT das mulheres da área rural (Tabela 1).

Tabela 1 - Perfil antropométrico de mulheres, segundo a faixa etária e localidade no Município de Barcelos- AM/1995-1996.

\begin{tabular}{|c|c|c|c|c|c|c|}
\hline \multirow{2}{*}{$\begin{array}{c}\text { NÃO } \\
\text { NUTRIZES } \\
\text { Faixa etária }\end{array}$} & \multicolumn{6}{|c|}{ VARIÁVEIS } \\
\hline & $\begin{array}{c}\text { PESO } \\
(\mathrm{kg})\end{array}$ & $\begin{array}{l}\text { ALTURA } \\
(\mathrm{cm})\end{array}$ & $\begin{array}{c}\text { IMC } \\
\left(\mathrm{kg} / \mathrm{m}^{2}\right)\end{array}$ & $\begin{array}{l}\mathrm{PCT} \\
(\mathrm{mm})\end{array}$ & $\begin{array}{l}\text { CB } \\
(\mathrm{cm})\end{array}$ & $\begin{array}{l}\text { CMB } \\
(\mathrm{cm})\end{array}$ \\
\hline URBANA & Média e dp & Média e dp & Média e dp & Média e dp & Média e dp & Média e dp \\
\hline$<20(n=9)$ & $50,4 \pm 11,8$ & $147,9 \pm 5,5$ & $22,9 \pm 4,6$ & $24,0 \pm 10,2$ & $27,1 \pm 3,9$ & $19,5 \pm 2,4$ \\
\hline $20-29(n=46)$ & $53,7 \pm 7,9$ & $151,0 \pm 5,4$ & $23,6 \pm 3,5$ & $19,3 \pm 6,7$ & $26,8 \pm 2,6$ & $20,7 \pm 2,1$ \\
\hline $30-39(n=36)$ & $55,3 \pm 11,1$ & $148,0 \pm 4,8$ & $25,2 \pm 4,7$ & $17,1 \pm 6,2$ & $27,3 \pm 3,3$ & $21,9 \pm 2,7$ \\
\hline$\geq 40(n=33)$ & $54,3 \pm 11,6$ & $147,7 \pm 5,1$ & $24,9 \pm 5,1$ & $12,6 \pm 2,1$ & $29,3 \pm 4,4$ & $22,9 \pm 3,4$ \\
\hline TOTAL $(n=124)$ & $54,1 \pm 10,2$ & $149,0 \pm 5,3$ & $24,4 \pm 4,4$ & $19,2 \pm 7,0$ & $27,5 \pm 3,4$ & $21,5 \pm 2,8$ \\
\hline \multicolumn{7}{|l|}{ RURAL } \\
\hline$<20(n=1)$ & 50,0 & 151,0 & 23,4 & 14,8 & 28,0 & 23,4 \\
\hline $20-29(n=8)$ & $48,2 \pm 6,1$ & $148,3 \pm 3,4$ & $21,9 \pm 2,3$ & $13,6 \pm 5,0$ & $26,7 \pm 2,2$ & $22,4 \pm 2,0$ \\
\hline $30-39(n=11)$ & $52,9 \pm 8,7$ & $148,6 \pm 4,8$ & $24,0 \pm 4,0$ & $14,7 \pm 7,5$ & $26,1 \pm 2,7$ & $21,4 \pm 1,3$ \\
\hline$\geq 40(n=5)$ & $49,0 \pm 6,0$ & $148,8 \pm 9,0$ & $22,4 \pm 3,3$ & $20,3 \pm 6,9$ & $26,3 \pm 2,2$ & $22,4 \pm 2,8$ \\
\hline $\operatorname{TOTAL}(\mathrm{n}=25)$ & $50,4 \pm 7,3$ & $148,6 \pm 5,7$ & $23,0 \pm 10,9$ & $13,8 \pm 5,2$ & $26,4 \pm 2,3$ & $22,1 \pm 1,9$ \\
\hline p valor* & 0,09 & 0,73 & 0,15 & $\mathrm{P}<0,05$ & 0,19 & 0,41 \\
\hline \multicolumn{7}{|l|}{ NUTRIZ } \\
\hline$(n=26)$ & $52,6 \pm 7,3$ & $147,7 \pm 5,3$ & $24,0 \pm 2,7$ & $18,6 \pm 6,6$ & $26,7 \pm 2,4$ & $20,9 \pm 1,7$ \\
\hline
\end{tabular}

* $p$ valor na comparação entre as médias dos dados antropométricos de mulheres da zona urbana e rural 
Na Tabela 2 é mostrada a prevalências das diferentes classificações nutricionais, de acordo com a faixa etária e procedencia. Verificou-se um percentual considerável de sobrepeso entre as mulheres residentes na zona urbana com idade entre 20 e 29 anos $(32,6 \%)$ e $>40$ anos $(36,4 \%)$.

Nas mulheres da área rural, constatouse uma prevalência de $4 \%$ para a obesidade e baixo peso e de $16 \%$ para sobrepeso (Tabela 2).
A distribuição do IMC por categorias é exibida em relação ao número total de mulheres residentes na zona rural $(\mathrm{n}=25)$ e ao numero de nutrizes estudadas $(n=26)$, dado que o tamanho da amostra dos dois grupos quando dividida por faixa etária se torna extremamente pequena. Verificou-se que 9 nutrizes $(34,6 \%)$ apresentaram-se com sobrepeso, não havendo mulheres com baixo peso ou obesidade (Tabela 2).

Tabela 2 - Estado nutricional (IMC) de mulheres, segundo a faixa etária e localidade no município de Barcelos, AM. 1995-1996.

\begin{tabular}{|c|c|c|c|c|c|c|c|c|}
\hline $\begin{array}{c}\text { NÃO } \\
\text { NUTRIZ }\end{array}$ & \multicolumn{8}{|c|}{ Faixa Etária } \\
\hline \multirow[t]{2}{*}{ URBANA } & \multicolumn{2}{|c|}{ Baixo Peso } & \multicolumn{2}{|c|}{ Normal } & \multicolumn{2}{|c|}{ Sobrepeso } & \multicolumn{2}{|c|}{ Obesidade } \\
\hline & $\mathrm{n}$ & $\%$ & $\mathrm{n}$ & $\%$ & $\mathrm{n}$ & $\%$ & $\mathrm{n}$ & $\%$ \\
\hline$<20$ & 1 & $(11,1)$ & 6 & $(66,7)$ & 1 & $(11,1)$ & 1 & $(11,1)$ \\
\hline $20-29$ & 3 & $(6,5)$ & 27 & $(58,7)$ & 15 & $(32,6)$ & 1 & $(2,2)$ \\
\hline $30-39$ & 1 & $(2,8)$ & 19 & $(52,8)$ & 7 & $(19,4)$ & 9 & $(25,0)$ \\
\hline$>40$ & 3 & $(9,1)$ & 15 & $(45,5)$ & 12 & $(36,4)$ & 3 & (9.1) \\
\hline$n=124$ & 8 & $(6,5)$ & 67 & $(54,0)$ & 35 & $(28,2)$ & 14 & $(11,3)$ \\
\hline \multicolumn{9}{|l|}{ RURAL } \\
\hline$n=25$ & 1 & $(4,0)$ & 19 & $(76,0)$ & 4 & $(16,0)$ & 1 & $(4,0)$ \\
\hline \multicolumn{9}{|l|}{ NUTRIZ } \\
\hline$n=26$ & 0 & (0) & 17 & $(65,4)$ & 9 & $(34,6)$ & 0 & $(0)$ \\
\hline
\end{tabular}

\section{DISCUSSÃO}

A avaliação do estado nutricional de grupos populacionais pode ser medida por parâmetros antropométricos, clínicos, bioquímicos e dietéticos. No presente estudo optou-se pelas variáveis antropométricas, dado o seu baixo custo operacional, facilidade e rapidez na execução, por não serem invasivas e, principalmente, pela sua aplicabilidade em estudos populacionais (Anselmo \& Burini,1991). Muitas revisões têm demonstrado as vantagens e desvantagens da utilização da antropometria como método para avaliação nutricional (Anselmo,1991; Navarro $\&$ Marchini, 2000). São referidas como limitações a baixa sensibilidade e especificidade em detectar distúrbios nutricionais específicos, como também não diferenciar o excesso de peso por edema daquele da obesidade (Santos, 1993;
OMS,1995). No entanto, a antropometria é o método mais utilizado como indicador do estado e das condições nutricionais em estudos epidemiológicos, principalmente pelas suas vantagens amplamente citadas.

Constatou-se que os valores encontrados em relação a altura de adultos femininos (148 e $149 \mathrm{~cm})$ foram muito próximos aos encontrados em estudos anteriores na população do Rio Negro, onde as mulheres apresentaram uma altura média de 145 cm (Giugliano et al.,1984), aos do Rio Solimões $(150 \mathrm{~cm})$ (Giugliano et al., 1981), como também da cidade de Manaus $(151 \mathrm{~cm})$ (Yuyama \& Nagahama, 1992). Os resultados obtidos evidenciaram que a estatura das mulheres amazonenses, possivelmente, seja uma das mais baixas do Brasil, quando confrontada com estudos realizados na região do $\mathrm{NE}(150 \mathrm{~cm})$, onde a desnutrição é predominante 
(ICNND,1965), e quando comparadas com grupos étnicos semelhantes, como o de Xavantes de 2 localidades do Mato Grosso $(154,6 \mathrm{~cm}$ e $155,3 \mathrm{~cm})$ (Gugelmin \& Santos, 2001). Tais dados significam, portanto, um resultado preocupante em razão da baixa estatura ser um fator de risco para a ocorrência de baixo peso do concepto ao nascer, como foi demonstrado por alguns pesquisadores (Ciari Jr. et al.,1975 e Siqueira et al., 1975).

Os valores médios de peso encontrados neste estudo foram semelhantes aos verificados por Yuyama \& Nagahama (1992) em mulheres em idade fértil $(53,6 \mathrm{~kg})$ e nutrizes $(52,0 \mathrm{~kg})$ de um bairro pobre de Manaus.

O IMC atingiu os limites máximos da normalidade em mulheres da área rural $(23,0 \mathrm{~kg})$ $\left.\mathrm{m}^{2}\right)$ e urbana $\left(24,0 \mathrm{~kg} / \mathrm{m}^{2}\right)$. Verificou-se uma diminuição no percentual das mulheres da área urbana com IMC considerado normal conforme o aumento da idade $(58,7 \% ; 52,8 \% ; 45,5 \%)$, o que pode ser explicado por fatores biológicos, que ocorrem de forma diferenciada ao longo da vida, somando a diminuição de gasto calórico devido à redução de atividades físicas. Esta tendência de evolução ponderal em função da idade também foi reportada em outros grupos populacionais (Coutinho et al., 1991; Ell et al.,1999; Martins et al.,1999). Os valores médios de IMC encontrados nas mulheres da área rural em todas as faixas etárias foram compatíveis com o padrão de normalidade estabelecido pela OMS (1995), já na área urbana a média do IMC das mulheres na faixa entre 30-39 anos foi classificada como sobrepeso $\left(25,2 \mathrm{~kg} / \mathrm{m}^{2}\right)$. Esses dados podem ser explicados pela maior prevalência de obesidade em mulheres da área urbana ( $25 \%$ vs. 9,1\%). Em aspectos gerais, o percentual de obesidade das mulheres da área urbana se apresentou semelhante aos encontrados em outros grupos populacionais (Coutinho et al., 1991; Andreazza, 1993; Guedes et al., 1995; Oliveira et al., 1998)

Em relação ao baixo peso, tanto na área urbana quanto na rural, os resultados obtidos em mulheres não nutrizes $(6,5 \%$ e $4,0 \%$, respectivamente), foram menores que em outros estudos (Coutinho et al.,1991; Martins et al., 1999).
Valores de prega cutânea tricipital (PCT), que indica a reserva energética do indivíduo na forma de tecido adiposo periférico, evidenciou que as moradoras da área urbana tinham os valores médios em torno de $19 \mathrm{~mm}$, estando, portanto, acima do parâmetro de normalidade $(16,5 \mathrm{~mm})$. A população feminina da área rural, em todas as faixas etárias, apresentou $o$ valor médio expressivamente inferior (+ 13,8 mm.), indicando uma diferença estatisticamente significativa entre as populações, provavelmente em decorrência da sobrevivência diária destas mulheres, a qual está estreitamente ligada à atividade braçal. O presente estudo revelou que as mulheres da área urbana de Barcelos apresentaram valores de PCT superiores àqueles obtidos em dados nacionais (Faintuch et al., 1983; Costa et al., 1987; Anselmo et al., 1992; Yuyama \& Nagahama,1992; Oliveira et al., 1998). Há evidências na literatura de uma possível influência regional, ditada pela desigualdade das condições sócio-econômicas.

Os valores obtidos para CB., especialmente das mulheres da área rural, foram semelhantes a outros estudos relatados (Faintuch et al.,1983; Costa et al, 1987; Anselmo et al.,1992; Yuyama \& Nagahama, 1992).

Outro componente da avaliação do estado nutricional da população estudada foi a circunferência muscular braquial (CMB) que estima a massa muscular total do corpo, viabilizando assim uma medida direta da reserva protéica, portanto um parâmetro no diagnóstico da desnutrição protéica do indivíduo (Anselmo \& Burini,1991; Paiva \& Campana,1992). Os resultados obtidos no presente estudo foram inferiores ao padrão preconizado por Jelliffe (1966) de 23,2 mm, exceto para o valor da faixa etária menor de 20 anos da zona rural, na qual o número da amostra estudada não foi numericamente representativo. Entretanto são semelhantes aos valores encontrados em outros estudos realizados no Amazonas (Yuyama \& Nagahama, 1992; Oliveira et al, 1998), e em outros estudos realizados em diferentes localidades fora da Amazônia (Faintuch et al., 1983; Costa et al., 1987). 
Em uma revisão procedida por Paiva \& Campana (1992), constatou-se que em grupos populacionais mais pobres, os valores da $\mathrm{CMB}$ são maiores, diferentemente do peso e da PCT, devido à hipertrofia muscular conseqüente da maior atividade física. Essa suposição poderia explicar também os resultados observados neste estudo.

A falta de dados de referência limita a aplicação da antropometria nas nutrizes (OMS, 1995). Os valores médios de IMC deste grupo encontraram-se no limite superior de normalidade, e em função do pequeno número amostral, os resultado de PCT, CB e CMB não têm significado epidemiológico expressivo. Os valores de $\mathrm{PCT}, \mathrm{CB}$ e $\mathrm{CMB}$ das nutrizes foram maiores que os encontrados por Yuyama \& Nagahama (1992).

As variações de peso ocorrem, principalmente, nos três primeiros meses de lactação, onde as perdas de peso são mais altas e mais lentas e se estabilizam posteriormente. Portanto para maior precisão dos dados de avaliação nutricional das nutrizes é importante a informação da duração e intensidade da amamentação no momento do estudo.

\section{CONCLUSÕES}

A estatura média registrada em mulheres do Município de Barcelos pode ser considerada baixa quando confrontada com outras regiões. Em relação à massa corpórea, apesar da maioria se situar dentro do limite máximo de normalidade, há grupos de risco que necessitam de um acompanhamento ou orientação quanto ao seu estado nutricional. As moradoras da área urbana apresentaram reserva adiposa maior que o parâmetro de normalidade, indicando menor atividade braçal que as moradoras da área rural.

\section{AGRADECIMENTOS}

Ao Dr Toby Vincent Barret pela revisão do abstract e ao FINEP pelo suporte financeiro.

\section{BIBLIOGRAFIA CITADA}

Alencar, F.H.; Yuyama, L.K.O.; Nagahama, D. 2000. Estudo antropométrico da população infantil da Calha do Rio Negro,
Amazonas, Brasil. III. Parque Nacional do Jaú. Acta Amazonica, 30(4): 665-9.

Andreazza, R. 1993 Avaliação nutricional de gestantes e sua relação com o peso do recém-nascido. Dissertação de mestrado da Escola Paulista de Medicina, São Paulo, SP. $118 p$

Anselmo, M.A.C.; Burini, R.C. 1991. Antropometria: aspectos históricos e visão crítica. Cadernos de Nutrição SBAN, 3:11-25.

Anselmo, M.A.C.; Burini, R.C.; Angeleli, A.Y.O.; Mota, N.G.S.; Campana, A.O. 1992. Avaliação do estado nutricional de indivíduos adultos sadios de classe média. Ingestão energética e protéica, antropometria, exames bioquímicos de sangue e testes de imunocompetência. Rev. Saúde Públ., S.Paulo, 26 (1): 46-53.

Campana, A. O.; Burini, R.C.; Anselmo, M.A.C. 1987. Population surveys in Brazil. Data on energy and protein intakes and on anthropometrics measurements of adult people. Wld. Rev. Nutr. Diet., 53: 209-34.

Ciari Jr. C; Almeida, P.A.M.; Siqueira, A.A.F. 1975. Relação entre o peso da criança ao nascer, altura materna, idade gestacional e restrição alimentar em gestantes normais. Rev. Saúde Públ., S. Paulo, 9 : 33-42.

Coitinho, D.C.; Leão, M.M.; Recine, E.; Sichieri, R. 1991. Condições nutricionais da população brasileira: adultos e idosos - Pesquisa Nacional sobre Saúde e Nutriçào. INAN: Instituto Nacional de Alimentação e Nutrição, Ministério da Saúde. Brasília.

Costa, O. L.; Santos, D. M.; Néspoli, C. A.; Centroducatte, F.; Souza, E.F.; Lima, E. G.; Faintuch, J. 1987. Padrões de normalidade para medidas antropométricas. Estudo sistemático em uma população adulta brasileira. Rev. Hosp.Clin. Fac. Med. S.Paulo, 42 (2): 49-54.

Diniz, D.B. 1991. Perfil nutricional de crianças de 2 a 6 anos de idade residentes na área urbana do município de Nova Olinda do Norte (AM). Dissertação de Mestrado, 
Universidade do Amazonas, Manaus, AM. 60p.

Ell, E.; Camacho, L.A.B.; Chor, D. 1999. Perfil antropométrico de funcionários de banco estatal no Estado do Rio de Janeiro/Brasil: I - índice de massa corporal e fatores sócio-demográficos. Cad. Saúde Pública, Rio de Janeiro, 15 (1): 113-121.

Faintuch, J.; Pereira, M.L.; Faintuch, B. L.; Machado, M.C.C.; Pinotti, H.W. 1983. Novos parâmetros para avaliação nutricional. - Investigação preliminar. Rev.Hosp. Clin. Fac. Med. S.Paulo, 38 (4): 164-66.

Fundação Instituto Brasileiro de Geografia e Estatística (FIBGE).1977. Estudo Nacional da Despesa Familiar (ENDEF). Consumo alimentar - Antropometria. Dados preliminares v.1, t.1. Rio de Janeiro.

Giugliano, R.; Giugliano, L.; Shrimpton, R. 1981. Estudos nutricionais das populações rurais da Amazônia. I. Várzea do Rio Solimões. Acta Amazonica, 11,(4): 773881.

Giugliano, R.; Shrimpton, R.; Marinho, H.A.; Giugliano, L. 1984. Estudos nutricionais das populações da Amazônia. II. Rio Negro. Acta Amazonica, 14 (3-4): 427-449.

Guedes, M.C.; Furumoto, R.; Ypiranga, L. 1995. Avaliação nutricional em populações: perfil antropométrico de usuários de um centro comercial de Brasília, DF. Rev. Saúde Dist. Fed., 6

(4): 42-7.

Guedes, M.R. 1989. Contribuição ao conhecimento do estado nutricional e renda familiar de gestantes sadias, em relação com seus recém-nascidos. Dissertação de Mestrado, INPA/FUA. Manaus, AM. 106p.

Gugelmin, S.A.; Santos, R.V. 2001 Ecologia humana e antropometria nutricional de adultos Xavantes, Mato Grosso, Brasil. Cad. Saúde Pública, Rio de Janeiro, 17 (2): 313-22.

Interdepartamental Commitee on Nutrition for National Development (ICNND) 1965. Northeast Brazil. Nutrition Survey. Washington. 293p.
Jelliffe, D.B. 1966 The assessment of the nutritional status of the community. Monogr. n 53 (WHO, Geneve) apud Campana, A. O. et al. 1987 Population surveys in Brazil: data on energy and protein intakes and on anthropometrics measurements of adult people. Wld. Rev. Nutr. Diet., 53: 209-34.

Lehti, K.K. 1989. Iron, folic acid and zinc intakes and status of low socio-economic pregnant and lactating Amazonian women. Eur. J. Clin. Nutr., 43:505-13.

Martins, I.S; Velásquez-Meléndez, G.; Cervato, A. M. 1999. Estado nutricional de grupamentos sociais da área metropolitana de São Paulo, Brasil. Cad. Saúde Pública, Rio de Janeiro, 15 (1): 71-78.

Nagahama, D.; Marinho, H.A.; Rocha, Y.R.; Ferraroni, M.J.R.; Silva, N.B.; Castro, J.S.; Onety, J.A. 1990. Avaliação nutricional e alimentar de pré-escolares de uma creche de Manaus e a influência da entidade no estado nutricional de sua população. Acta Amazonica, 20(único): 119-28.

Navarro, A. M.; Marchini, J.S. 2000. Uso de medidas antropométricas para estimar gordura corporal em adultos. Nutrire: Rev. Soc. Brás. Alim. Nutr. São Paulo, SP, 19/ 20: 31-47.

Oliveira, M.C.; Nagahama, D.; Mourão, M.P.G.; Motta, G.C.; Nogueira, J.M.S.; Nascimento, M.R.; Magalhães, S.M.M.; Pinto, V.C.R.; Souza, M.A.; Pereira Jr.,E.A.P. No prelo. Parâmetros antropométricos para diagnóstico nutricional em ambulatório. Rev. UA, Série Ciências da Saúde, $6(1 / 2)$

Organização Mundial da Saúde (OMS). 1995. El estado fisico: uso e interpretación de la antropometría. Informe de um Comité de Expertos de la OMS. Série de Informes Técnicos- 854.

Paiva, A.R.; Campana, A.O.O. 1992. Diagnóstico da desnutrição energéticoprotéica em pacientes adultos: antropometria. Caderno de Nutrição, 4: 27-37.

Santos, R.V. 1993. Crescimento físico e estado nutricional de populações indígenas brasileiras. Cad. Saúde Públ., Rio de Janeiro, 9 (supl.1): 46-57. 
SEBRAE/AM.1994. Diagnóstico sócioeconômico e cadastro empresarial de Barcelos. Manaus: Departamento de Estudo e Pesquisa, 1999p.

SEMSA/UNICEF. 1996. Diagnóstico das condições de saúde da mulher e da criança no Municipio de Manaus, Amazonas. Manaus, AM, 113p.

Silva, W. 1959. Inquérito sobre consumo de alimentos e nutrimentos, avaliação do estado nutritivo e situação econômica da população da Amazônia. Inquérito sobre as condições de alimentação e nutrição de gestantes e nutrizes da Amazônia. Bol. Com. Nac. Alim., 9 (2): 1-60.

Shrimpton, R. 1984. Food consumption and dietary adequacy according to income in 1200 families, Manaus, Amazonas, Brazil, 1973-1974. Arch. Latinoamer. Nutr. 34: 615-29.
Siqueira, A.A.F.; Ciari Jr., C.; Almeida, P.A.M.; Tanaka, A.C.A.; Montelone, P.P.R.; Arruda, J.J.G.; Delascio, D. 1975. Influência de altura e ganho de peso maternos e da idade gestacional sobre o peso do recém-nascido: estudo de três grupos de gestantes normais. Rev. Saúde Públ.S.Paulo, 9: $331-42$.

Yuyama, L.K.; Nagahama, D. 1992. Levantamento antropométrico de uma população feminina de um bairro pobre de Manaus, AM. Rev.UA. Série: Ciências da Saúde, Manaus, 1(1): 79-84.

Yuyama, L.K.O.; Aguiar, J.P.L.; Macedo, S.H.M.; Yonekura, L.; Nagahama, D.; Alencar, F.H. 1999. Perfil nutricional da dieta dos pré-escolares do Município de Nhamundá-AM. Acta Amazonica. 29 (4): 651-54 